$\mathbb{T}$ periodica polytechnica

Mechanical Engineering

$56 / 2(2012) 99+103$

doi: 10.3311/pp.me.2012-2.04

web: http://www.pp.bme.hu/me

(c) Periodica Polytechnica 2012

RESEARCH ARTICLE

\section{Stability of an elastic supported flat plate subjected to potential flow}

\author{
Zsolt Szabó / Ambrus Zelei / Gábor Stépán
}

Received 2012-06-30

\begin{abstract}
In this paper we investigated the dynamics of a thin, flat plate placed in potential planar flow and attached by springs to the wall. Furthermore, the centre of gravity of the plate was assumed to move only perpendicular to the direction of the flow. Thus, a two-degree-of-freedom oscillatory system was analysed, whose natural frequencies depend on the flow velocity.

The equations of motion were derived for two cases: first, the flow forces acting on the plate were determined using a quasisteady approach; in the second case, we used the formulas derived by Theodorsen assuming unsteady potential flow. Finally, we obtained and compared the stability charts for the two cases.
\end{abstract}

\section{Keywords}

potential flow $\cdot$ aerodynamic instability $\cdot$ flutter

\section{Acknowledgement}

This research was partially supported by the Hungarian Scientific Research Found OTKA Grant No. K72911, HAS-BUTE Research Group on Dynamics of program of the ÚMTF (New Hungary Development Plan).

\section{Zsolt Szabó}

HAS-BUTE Research Group on Dynamics of Machines and Vehicles, H-1111 Budapest, Múegyetem rkp. 5, Hungary

e-mail: szazs@mm.bme.hu

\section{Ambrus Zelei}

HAS-BUTE Research Group on Dynamics of Machines and Vehicles, H-1111 Budapest, Múegyetem rkp. 5, Hungary

e-mail: zelei@mm.bme.hu

\section{Gábor Stépán}

Department of Applied Mechanics, BUTE, H-1111 Budapest, Múegyetem rkp. 5 , Hungary

e-mail: stepan@mm.bme.hu

\section{Introduction}

The presented investigations were motivated by the participitation in a research project, whose aim was to develop a procedure to determine the so-called flutter derivatives of bridge sections. Knowing the functions of these derivatives helps to predict or avoid the aerodynamic instability caused by flutter. The phenomena of flutter, similarly to galloping, is a self-excited vibration occurring in the case of flexibly supported bodies (like slender bridges or cables) placed in some kind of steady flow [1],[2]. The other large group of stability problems are induced either by originally unsteady flow like buffeting or by such an unsteady flow that is caused primarily by the geometry of the body but not its motion, like in the case of vortex-shedding [2], [4].

In the following we consider a thin, flat, rectangular plate of length $l$ and width $B$. The homogeneous plate has a mass $m$ and its cross-section seen in Fig. 1 can perform only planar motion. The support of the plate is modelled by a vertically placed straight spring and a torsional one of stiffness $k_{h}$ and $k_{\alpha}$, respectively. The springs attached to the centre of gravity (i.e. the middle) of the plate are also assumed to have damping whose coefficients are $c_{h}$ and $c_{\alpha}$ in vertical and torsional directions, respectively. The angular velocity of the plate is perpendicular to the plane of the motion and the mass and the mass moment of inertia is denoted by $m$ and $J$, respectively.

The centre of gravity of the plate can move only vertically and its displacement is denoted by $h$. Thus, the model has only two degrees of freedom: the generalised coordinates are $q_{1}=h$ and the angle of the plate w.r.t. the horizontal plane, $q_{2}=\alpha$. (If the plate were the section of a bridge, $h$ and $\alpha$ would be related to its bending and torsion, respectively.) Furthermore, we assume that the plate is in equilibrium when $h=\alpha=0$, that is the effect of gravity is compensated by the initial elongation of the spring $k_{h}$.

Taking into consideration the previous assumptions, the equations of motion of the plate is described by

$$
\mathbf{M} \ddot{\mathbf{q}}+\mathbf{C}_{\mathrm{mech}} \dot{\mathbf{q}}+\mathbf{K}_{\mathrm{mech}} \mathbf{q}=\mathbf{Q}_{\text {flow }},
$$




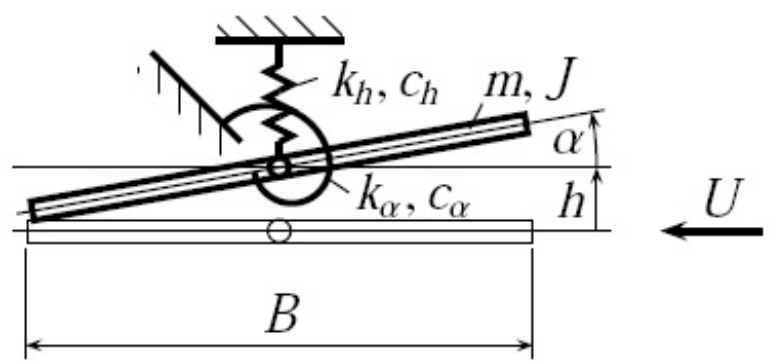

Fig. 1. Sketch of the flat plate model.

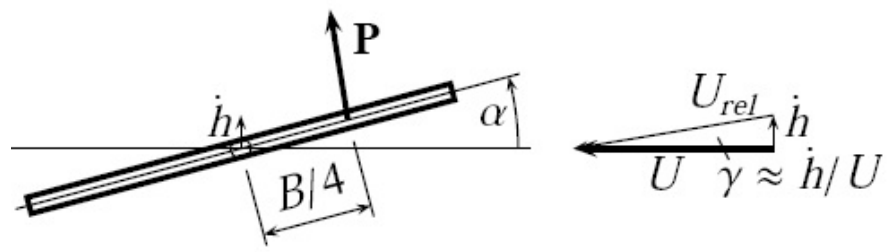

Fig. 2. The flow force acting on the plate.

where

$$
\mathbf{M}=\left[\begin{array}{cc}
m & 0 \\
0 & J
\end{array}\right], \mathbf{C}_{\text {mech }}=\left[\begin{array}{cc}
c_{h} & 0 \\
0 & c_{\alpha}
\end{array}\right], \mathbf{K}_{\text {mech }}=\left[\begin{array}{cc}
k_{h} & 0 \\
0 & k_{\alpha}
\end{array}\right]
$$

are the coefficient matrices of the mechanical parameters and $\mathbf{Q}_{\text {flow }}$ is the generalised force determined by the flow.

In the following we consider the flow forces for two different cases: in the next section a quasi-steady flow model is considered where the effect of the angular velocity of the plate is neglected; afterwards, we apply the derived theoretical flow forces acting on a thin, flat, infinite plate performing harmonic (transversal or rotational) oscillations in a potential planar flow.

\section{Quasi-steady potential flow}

According to the theory of potential planar flows, the reduced flow force acting on a section of length $l$ of a flat plate having vertical velocity $\dot{h}$ (see Fig. 2) is the following:

$$
\mathbf{P}=\left[\begin{array}{c}
-P \sin \gamma \\
P \cos \gamma
\end{array}\right], \quad P=\frac{\rho}{2} U_{r e l}^{2} l B \cdot 2 \pi \sin (\alpha-\gamma),
$$

where $U_{\text {rel }}$ is the relative flow velocity and $\alpha-\gamma \approx \alpha-\dot{h} / U$ is the angle of attack of $U_{r e l}$. That is, the flow force $\mathbf{P}$ is perpendicular to the relative flow velocity.

If we consider the angular velocity $\dot{\alpha}$, too, then the virtual power of the flow force yields

$$
\begin{gathered}
\mathbf{P} \cdot \delta \mathbf{v}_{B / 4} \equiv \mathbf{P} \cdot\left[\begin{array}{c}
-\frac{B}{4} \delta \dot{\alpha} \sin \alpha \\
\delta \dot{h}+\frac{B}{4} \delta \dot{\alpha} \cos \alpha
\end{array}\right] \equiv \\
P \frac{B}{4} \cos (\alpha-\gamma) \delta \dot{\alpha}+P \underbrace{\cos \gamma}_{U / U_{\text {rel }}} \delta \dot{h} .
\end{gathered}
$$

Thus, the generalised force $\mathbf{Q}_{\text {flow }}$ appearing on the right handside of Eq. (1) is as follows:

$$
\begin{array}{r}
\mathbf{Q}_{\text {flow }}=\frac{\rho}{2} U_{r e l}^{2} l B \cdot 2 \pi\left[\begin{array}{c}
\frac{U}{U_{\text {rel }}} \sin (\alpha-\gamma) \\
\frac{B}{8} \sin (2 \alpha-2 \gamma)
\end{array}\right] \approx \\
\frac{\rho}{2} l B \cdot 2 \pi\left[\begin{array}{c}
-U \dot{h}+U^{2} \alpha \\
-\frac{B}{4} U \dot{h}+\frac{B}{4} U^{2} \alpha
\end{array}\right],
\end{array}
$$

where we assumed that $U_{r e l} \approx U$ and $\alpha-\gamma$ is small.

\subsection{Linearised equations of motion}

For the case of small $\dot{h}$ and $\alpha$, the equations of motion can be linearised around $h=0$ and $\alpha=0$. Introducing $\eta=4 h / B$ and $\tau=t \omega_{1}\left(\omega_{1}=\sqrt{k_{h} / m}\right)$ Eq. 11$)$ can be rewritten in dimensionless form:

$$
\begin{aligned}
\eta^{\prime \prime}+2 \zeta_{1} \eta^{\prime}+\eta & =4 \mu\left(-u \eta^{\prime}+4 u^{2} \alpha\right), \\
\alpha^{\prime \prime}+2 \zeta_{2} \varepsilon \alpha^{\prime}+\varepsilon^{2} \alpha & =4 \vartheta\left(-u \eta^{\prime}+4 u^{2} \alpha\right)
\end{aligned}
$$

where $^{\prime}=\mathrm{d} / \mathrm{d} \tau$, furthermore, the dimensionless parameters are defined as follows:

$$
\zeta_{1}=\frac{c_{h}}{2 \sqrt{m k_{h}}}, \quad \zeta_{2}=\frac{c_{\alpha}}{2 \sqrt{J k_{\alpha}}}, \quad \varepsilon=\frac{\omega_{2}}{\omega_{1}} \equiv \sqrt{\frac{m k_{\alpha}}{J k_{h}}},
$$

and

$$
\begin{array}{r}
u=\frac{U}{B \omega_{1}}, \quad \mu=\frac{\rho l B^{2} \pi}{4 m}, \quad \vartheta=\frac{\rho l B^{4} \pi}{64 J} \equiv \mu \beta^{2}, \\
\beta=\frac{B}{4 r_{i}}, \quad r_{i}=\sqrt{\frac{J}{m}} .
\end{array}
$$

\subsection{Stability analysis}

Arranging the equations of motion of (Eq. 6) in matrix form again, we obtain

$$
\mathbf{q}^{\prime \prime}+2\left[\begin{array}{cc}
\zeta_{1}+2 \mu u & 0 \\
2 \vartheta u & \zeta_{2} \varepsilon
\end{array}\right] \mathbf{q}^{\prime}+\left[\begin{array}{cc}
1 & -16 \mu u^{2} \\
0 & \varepsilon^{2}-16 \vartheta u^{2}
\end{array}\right] \mathbf{q}=\mathbf{0},
$$

where $\mathbf{q}=(\eta, \alpha)$ is the vector of the dimensionless generalised coordinates and $u$ is the dimensionless flow velocity defined in (8).

The characteristic polynom of Eq. 99 is

$$
\left|\begin{array}{cc}
\lambda^{2}+2\left(\zeta_{1}+2 \mu u\right) \lambda+1 & -16 \mu u^{2} \\
4 \vartheta u \lambda & \lambda^{2}+2 \zeta_{2} \varepsilon \lambda+\varepsilon^{2}-16 \vartheta u^{2}
\end{array}\right| \equiv
$$

where

$$
\begin{aligned}
& a_{3}=2\left(\zeta_{2} \varepsilon+\zeta_{1}+2 \mu u\right), \\
& a_{2}=1+\varepsilon^{2}-16 \vartheta u^{2}+4\left(\zeta_{1}+2 \mu u\right) \zeta_{2} \varepsilon, \\
& a_{1}=2\left(\zeta_{2} \varepsilon+\left(\zeta_{1}+2 \mu u\right) \varepsilon^{2}-16 \vartheta u^{2} \zeta_{1}\right), \\
& a_{0}=\varepsilon^{2}-16 \vartheta u^{2} .
\end{aligned}
$$




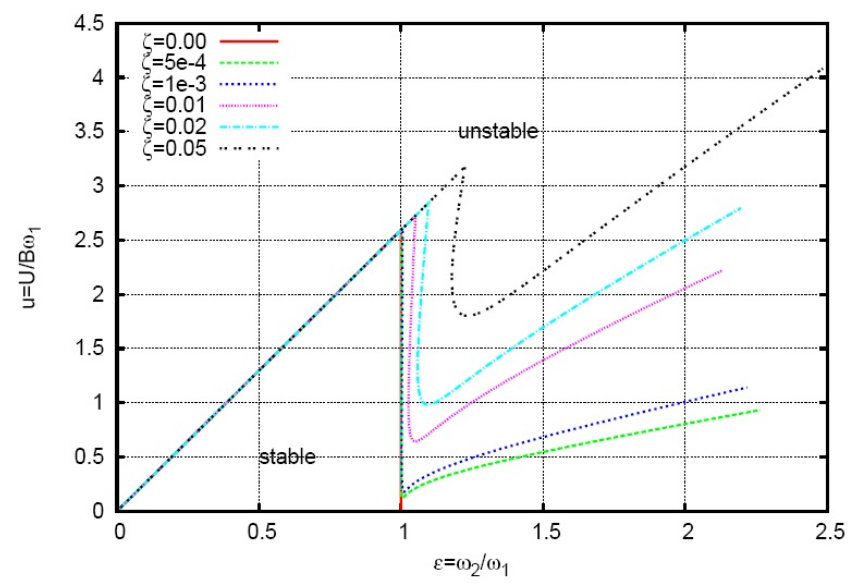

Fig. 3. The stability boundaries for the quasi-steady model.

The necessary and satisfactory condition of asymptotic stability (i.e. $\operatorname{Re} \lambda_{i}<0, \forall i$ ) is that the minors of the Hurwitz determinant are positive:

$$
H_{i}=\left|\begin{array}{cccccc}
a_{0} & 0 & 0 & 0 & \cdots & 0 \\
a_{2} & a_{1} & a_{0} & 0 & \ldots & 0 \\
a_{4} & a_{3} & a_{2} & a_{1} & \ldots & 0 \\
\vdots & \vdots & \vdots & \ddots & & \vdots \\
a_{2 i} & a_{2 i-1} & a_{2 i-2} & a_{2 i-3} & \cdots & a_{i}
\end{array}\right|>0,
$$

In our case: $H_{0} \equiv a_{0}, H_{i}=a_{0} \tilde{H}_{i}$, and $H_{4}=H_{3}$. Thus, the following inequalities must hold:

$$
\begin{aligned}
a_{0}>0 & \Rightarrow u<u_{c r, 0} \equiv \frac{\varepsilon}{\sqrt{16 \vartheta}} \equiv \frac{\varepsilon}{4 \beta \sqrt{\mu}}, \\
a_{1}>0 & \Rightarrow \ldots, \\
a_{1} a_{2}-a_{3} a_{0} \equiv \tilde{H}_{2}>0 & \Rightarrow \ldots, \\
a_{3} \tilde{H}_{2}-a_{1}^{2} \equiv \tilde{H}_{3}>0 & \Rightarrow f(\varepsilon, u)>0 .
\end{aligned}
$$

One can also show that it is sufficient to satisfy the inequalities (Eq. 13a and (Eq. $13 \mathrm{~d}$, i.e. $a_{1}>0$ and $\tilde{H}_{2}>0$ are weaker conditions.

The critical flow velocity in SI dimension expressed from (Eq. 13a with the original system parameters is

$$
U_{c r, 0}=\frac{2}{B} \sqrt{\frac{k_{\alpha}}{\rho l \pi}} .
$$

The boundary of the stability domain on the dimensionless $\varepsilon-u$ plane is determined by

$$
u_{c r}(\varepsilon)=\min \left(u_{c r, 0}(\varepsilon), u_{c r, H_{3}}(\varepsilon)\right),
$$

where $u_{c r, H_{3}}$ is obtained numerically from $f\left(\varepsilon, u_{c r, H_{3}}\right)=0$. The boundary curves for various $\zeta=\zeta_{1}=\zeta_{2}$ parameters can be seen in Fig. 3. The straight line represents $u_{c r, 0}(\varepsilon)$ and the curves above $\varepsilon=1$ belong to $u_{c r, H_{3}}(\varepsilon)$.

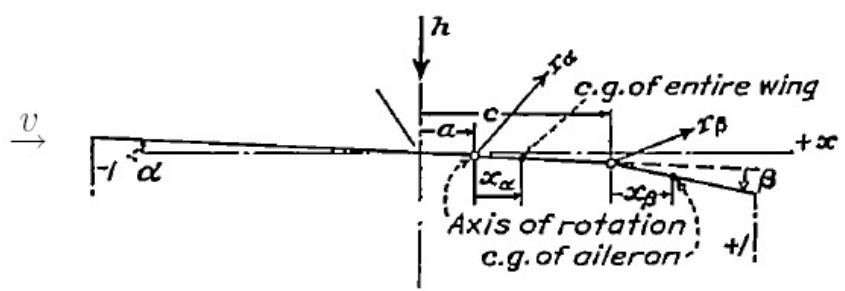

Fig. 4. The sketch of a wing with aileron investigated by THEOdorsen

\section{Unsteady potential flow}

\subsection{Theodorsen's model}

THeodorsen [7] investigated a thin infinite wing with aileron (see Fig. 4) and derived the flow forces acting on it for the case when the wing carries out harmonic oscillations in vertical and rotational directions, respectively. In his model the upwind side was to the left and the wind velocity was noted by $v$. Furthermore, $\alpha$ and $h$ were assumed to be positive in the clockwise and downwards directions, respectively, and the geometry was dimensionless with the half chord $b$ as reference unit. If we choose $a$ (the axis of rotation), $x_{a}$ (the centre of gravity of the entire wing), and $\beta$ (the angle of the aileron) to zero, then we obtain a similar geometry shown in Fig. 1 .

After long analytical calculations using velocity potentials and determining the circulation, the aerodynamic forces and moments can be expressed. Applying $a=x_{a}=\beta=0$, the frequency-dependent force acting in the middle of a wing section of length $l$ in the direction of $h$ is

$$
P=-\rho l b^{2}(v \pi \dot{\alpha}+\pi \ddot{h})-2 \pi \rho l v b C\left(v \alpha+\dot{h}+\frac{b}{2} \dot{\alpha}\right),
$$

and the frequency-dependent moment acting in the direction of $\alpha$ is

$$
M=-\rho l b^{2}\left(\pi v \frac{b}{2} \dot{\alpha}+\pi \frac{b^{2}}{8} \ddot{\alpha}\right)+\rho l v b^{2} \pi C\left(v \alpha+\dot{h}+\frac{b}{2} \dot{\alpha}\right),
$$

where $C=C(k)$ is a complex function of $k=b \omega / v$, the dimensionless frequency of oscillation.

In order to get the expressions for the model in Fig. 11 first of all we have to substitute $v=U$ and $b=B / 2$. Afterwards, if we rotate Theodorsen's model with $180^{\circ}$, the upwind side and direction of $h$ will be identical to those of our models except the direction of $\alpha$. That is, we have to change the sign of $\alpha$-s in Eq. (16) to get the flow force acting on our flat plate when it carries out harmonic oscillations. Similarly, we also have to change the sign of $h$-s in Eq. (17). Finally, we get

$$
\begin{aligned}
P= & \frac{\rho}{2} l B 2 \pi\left\{\frac{B}{4}(U \dot{\alpha}-\ddot{h})+C(k)\left(U^{2} \alpha-U \dot{h}+\frac{B}{4} U \dot{\alpha}\right)\right\} \\
M= & \frac{\rho}{2} l B 2 \pi\left\{\frac{B}{4}\left(-\frac{B}{4} U \dot{\alpha}-\frac{B^{2}}{32} \ddot{\alpha}\right)+\right. \\
& \left.\frac{B}{4} C(k)\left(U^{2} \alpha-U \dot{h}+\frac{B}{4} U \dot{\alpha}\right)\right\}
\end{aligned}
$$




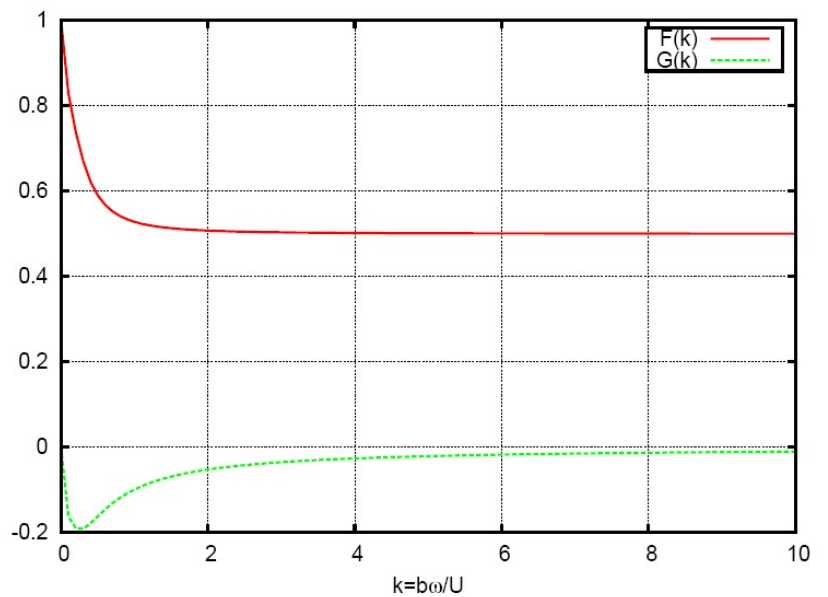

Fig. 5. The graphs of $F(k)$ and $G(k)$.

\subsection{The complex $C(k)$ function}

The complex $C(k)$ function introduced in Eqs. [16)-(17) is defined as follows:

$$
C(k)=\frac{\int_{1}^{\infty} \frac{x}{\sqrt{x^{2}-1}} e^{-i k x} d x}{\int_{1}^{\infty} \frac{x+1}{\sqrt{x^{2}-1}} e^{-i k x} d x},
$$

which can be expressed by Bessel functions of the first and second kind [2]. Moreover, according to Starossek [4], it is approximately

$$
C(k) \approx 1-\frac{0.165 k}{k-0.0455 i}-\frac{0.335 k}{k-0.3 i} \equiv F(k)+i G(k),
$$

where

$$
\begin{aligned}
& F(k) \approx 1-\frac{0.165 k^{2}}{k^{2}+0.0455^{2}}-\frac{0.335 k^{2}}{k^{2}+0.09}, \\
& G(k) \approx-\frac{0.165 \cdot 0.0455 k}{k^{2}+0.0455^{2}}-\frac{0.335 \cdot 0.3 k}{k^{2}+0.09} .
\end{aligned}
$$

The graphs of $F(k)$ and $G(k)$ can be seen in Fig. 5

\subsection{The flutter derivatives}

The interpretation of the complex $C(k)$ function can be revealed if we assume the generalised coordinates as complex functions of time. Since the derivation of the flow forces was carried out for harmonic transversal and rotational motions, this means

$$
q_{i}(t)=q_{i}^{0} e^{i \omega t}, \quad \dot{q}_{i}(t)=i \omega q_{i}(t), \quad \ddot{q}_{i}(t)=-\omega^{2} q_{i}(t) .
$$

Thus,

$$
\begin{aligned}
& C(k) q_{i}(t)=F(k) q_{i}+\frac{G}{\omega} i \omega q_{i} \equiv F(k) q_{i}+\frac{G}{\omega} \dot{q}_{i}, \\
& C(k) \dot{q}_{i}(t)=F(k) \dot{q}_{i}-\omega G(k) q_{i}\left(\equiv F(k) \dot{q}_{i}+\frac{G}{\omega} \ddot{q}_{i}\right) .
\end{aligned}
$$

Substituting these expressions in formulas (Eq. 18a) and (Eq. 18b), we obtain the generalised force vector from the flow:

$$
\begin{aligned}
\mathbf{Q}_{\text {flow }} \equiv & {\left[\begin{array}{c}
P \\
M
\end{array}\right]=\frac{\rho}{2} l B U \cdot 2 \pi\left[\begin{array}{cc}
-F & \frac{B}{4}\left(1+F+\frac{4 G}{K}\right) \\
-\frac{B}{4} F & \frac{B^{2}}{16}\left(-1+F+\frac{4 G}{K}\right)
\end{array}\right] \dot{\mathbf{q}} } \\
& +\frac{\rho}{2} l U^{2} \cdot 2 \pi\left[\begin{array}{cc}
\frac{K^{2}}{4}+K G & \frac{B}{4}(4 F-K G) \\
\frac{B}{4} K G & \frac{B^{2}}{16}\left(4 F-K G+\frac{K^{2}}{8}\right)
\end{array}\right] \mathbf{q},
\end{aligned}
$$

where $K=B \omega / U \equiv 2 k$ is another dimensionless form of the frequency of oscillation.

Finally, the equations of motion for the unsteady potential flow will have the following form:

$$
\begin{aligned}
\mathbf{M} \ddot{\mathbf{q}}+\mathbf{C}_{\text {mech }} \dot{\mathbf{q}}+\mathbf{K}_{\text {mech }} \mathbf{q}= & \frac{\rho l B \pi}{4} U\left[\begin{array}{cc}
H_{1} & \frac{B}{4} H_{2} \\
\frac{B}{4} A_{1} & \frac{B^{2}}{16} A_{2}
\end{array}\right] \dot{\mathbf{q}}+ \\
& \frac{\rho l \pi}{4} U^{2}\left[\begin{array}{cc}
H_{4} & \frac{B}{4} H_{3} \\
\frac{B}{4} A_{4} & \frac{B^{2}}{16} A_{3}
\end{array}\right] \mathbf{q},
\end{aligned}
$$

where the following coefficients were introduced (flutter derivatives):

$$
\begin{aligned}
& A_{1}=H_{1}=-4 F(k), \\
& H_{2}=4\left(1+F(k)+\frac{2 G(k)}{k}\right), \\
& A_{2}=4\left(-1+F(k)+\frac{2 G(k)}{k}\right), \\
& H_{4}=8 k G(k)+4 k^{2}, \\
& A_{4}=8 k G(k), \\
& H_{3}=16 F(k)-8 k G(k), \\
& A_{3}=16 F(k)-8 k G(k)+2 k^{2} .
\end{aligned}
$$

For arbitrary bridge sections, the flutter derivatives $H_{i}$ and $A_{i}$ can be determined experimentally or by numerical simulations (see [1], [6], and [5]). Then, the dynamic behaviour of the bridge can be predicted and the critical wind velocity can be calculated, as described in the followings, for example.

\subsection{Determining the stability boundaries}

The dimensionless form of the equations of motion will be similar to Eq. (9):

$$
\begin{array}{r}
\mathbf{q}^{\prime \prime}+\left[\begin{array}{cc}
2 \zeta_{1} & 0 \\
0 & 2 \zeta_{2} \varepsilon
\end{array}\right] \mathbf{q}^{\prime}+\left[\begin{array}{cc}
1 & 0 \\
0 & \varepsilon^{2}
\end{array}\right] \mathbf{q}= \\
=u\left[\begin{array}{ll}
\mu H_{1} & \mu H_{2} \\
\vartheta A_{1} & \vartheta A_{2}
\end{array}\right] \mathbf{q}^{\prime}+u^{2}\left[\begin{array}{cc}
\mu H_{4} & \mu H_{3} \\
\vartheta A_{4} & \vartheta A_{3}
\end{array}\right] \mathbf{q} .
\end{array}
$$

Putting $\mathbf{q}=\exp \lambda \tau$ into Eq. 27, we obtain the characteristic equation in the form of

$$
\begin{array}{r}
\left|\begin{array}{rr}
\lambda^{2}+\left(2 \zeta_{1}-\mu u H_{1}\right) \lambda+1 & -\mu u^{2} H_{4}-\mu u H_{2} \lambda-\mu u^{2} H_{3} \\
-\vartheta u A_{1} \lambda-\vartheta u^{2} A_{4} & \lambda^{2}+\left(2 \zeta_{2} \varepsilon-\vartheta u A_{2}\right) \lambda+\varepsilon^{2}-\vartheta u^{2} A_{3}
\end{array}\right| \equiv \\
\lambda^{4}+a_{3} \lambda^{3}+a_{2} \lambda^{2}+a_{1} \lambda+a_{0}=0,
\end{array}
$$

where

$$
a_{0}=\varepsilon^{2}-\left(\mu \varepsilon^{2} H_{4}+\vartheta A_{3}\right) u^{2}+\left(H_{4} A_{3}-H_{3} A_{4}\right) \mu \vartheta u^{4},
$$

for example.

Although Eq. (28) is valid only for $\lambda=i \omega$, the limit case $\omega \rightarrow 0$ (static stability loss) requires that $a_{0}$ should be zero.

Since

$$
\begin{aligned}
\omega \rightarrow 0 \Leftrightarrow k \rightarrow 0 \Rightarrow\left\{\begin{array}{c}
G(k) \rightarrow 0 \\
F(k) \rightarrow 1
\end{array}\right\} \Rightarrow & \left\{\begin{array}{c}
H_{3}, A_{3} \rightarrow 16 \\
H_{4}, A_{4} \rightarrow 0
\end{array}\right\} \Rightarrow \\
& a_{0} \rightarrow \varepsilon^{2}-16 \vartheta u^{2},
\end{aligned}
$$




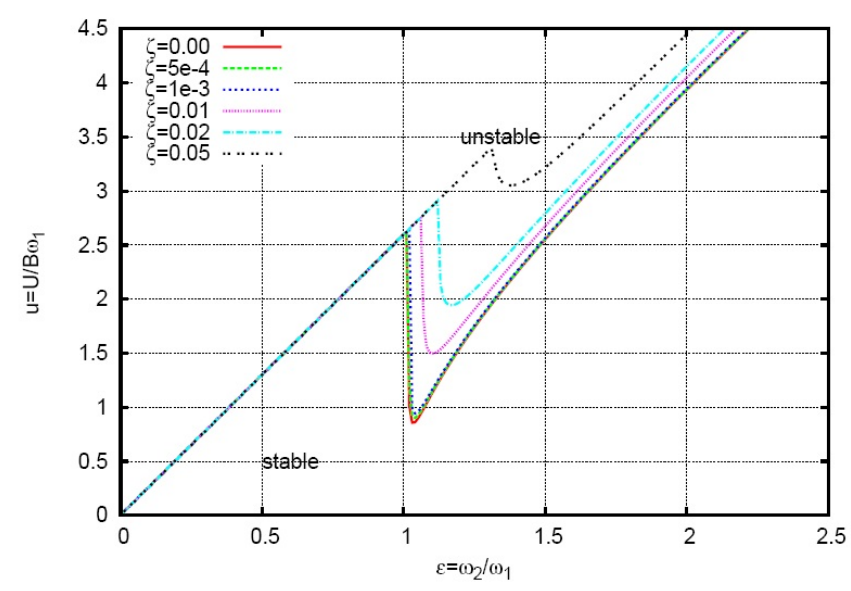

Fig. 6. The stability boundaries of the unsteady model.

we get that $a_{0}=0$ yields the same critical flow velocity as the one we obtained in Eq. 13a for the quasi-steady case.

Investigating the case $\omega>0$, the following two equations (obtained for the imaginary and real parts) must be satisfied simultaneously:

$$
\begin{aligned}
-a_{3}(\omega, u) \omega^{2}+a_{1}(\omega, u) & =0, \\
\omega^{4}-a_{2}(\omega, u) \omega^{2}+a_{0}(\omega, u) & =0 .
\end{aligned}
$$

Now, we have to fix some of the parameters (e.g. $\zeta_{1}, \zeta_{2}, \mu$, and $\vartheta$ ) and find the value pairs of others (e.g. $\varepsilon$ and $u$ ) for whom the equations (30)-31) are true.

A possible method for this if we determine $\omega_{0}$ for the case of $u=0$ from Eq. 30):

$$
\omega_{0}^{2}=\frac{\left.a_{1}(\omega, u)\right|_{u=0}}{\left.a_{3}(\omega, u)\right|_{u=0}} \equiv \frac{\zeta_{1} \varepsilon^{2}+\zeta_{2} \varepsilon}{\zeta_{1}+\zeta_{2} \varepsilon+\frac{\pi}{2}\left(\frac{\vartheta}{2} \zeta_{1}+\zeta_{2} \varepsilon\right)} .
$$

Then, changing $u$ with a small step, we can iterate to $\omega(u)$. Afterwards, we calculate $\tilde{\omega}$ from Eq. (31). In the next step, if the sign of $\tilde{\omega}-\omega$ has changed, we the decrease the step size and change its sign, too, and modify the value of $u$ with the new step and iterate this process until $\tilde{\omega} \approx \omega(u)$. The stability boundaries obtained by this procedure can be seen in Fig. 6. which are similar to the ones presented by KLÖPPEL and THIELE for $\varepsilon>1$ [3].

\section{Conclusions}

We have investigated a thin flat plate placed with an elastic support in a potential planar flow. It is well-known that the flow forces can cause flutter, which means that the system loses its stability through vibrations.

Two cases have been considered: in the simpler case the flow forces have been derived using quasi-steady assumption; in a more precise model we have used the frequency-dependent flow forces derived by Theodorsen taking into account the unsteadiness of the potential flow, but only for a harmonically oscillating plate. This represents the critical state between the decaying vibrations and the self-excited flutter.
The stability charts for the two cases are similar: if the natural frequency belonging to the rotating vibrational mode is less then the natural frequency of the transversal mode shape $\left(\varepsilon=\omega_{2} / \omega_{1}<1\right)$, then static stability loss occurs (when $U>2 \sqrt{k_{\alpha} / \rho l \pi} / B$ ) and flutter can occur only if $\varepsilon>1$, in both models. The difference is between the stability boundary for the flutter: the quasi-steady model yields lower critical values of the flow velocity for fixed $\omega_{2} / \omega_{1}$ ratio than the Theodorsen model.

\section{References}

1 Avşar Ö, Determination of flutter derivatives from free vibration test results, Master's thesis, Institut für Baustatik und Stahlbau, Technische Universität Hamburg-Harburg, 2003.

2 Borri C, Costa C, Bridge Aerodynamics and Aeroelastic Phenomena Chapter 1:Bridges, Wind Effects on Buildings and Design of Wind-Sensitive Structures (In Ted Stathopoulos e., ed.), CISM Courses and Lectures No. 493, Springer Wien New York, 2007.

3 Klöppel K, Thiele F, Modellversuche im Windkanal zur Bemessung von Brücken gegen die Gefahr winderregter Schwingungen, Der Stahlbau 32 (1967), no. 12, 353-365.

4 Starossek U, Brückendynamik -Winderregte Schwingungen von Seilbrücken, Vieweg, 1992.

5 Szabó G, Györgyi J, Numerical simulation of the flutter performance of different generic bridge cross sections, Periodica Polytechnica, Ser. Civil Eng. 55 (2011), no. 2, 137-146, DOI 10.3311/pp.ci.2011-2.06.

6 Szabó G, Kristóf G, Three-dimensional numerical flutter simulation, In The 5th International Symposium on Computational Wind Engineering (CWE2010), Chapel Hill, North Carolina, USA, 2010.

7 Theodorsen T, General theory of aerodynamic instability and the mechanism of flutter (1935). 